$\triangle$ CTA $\mathbb{N E O P H I L O L O G I C A}$

DOI: 10.4312/an.48.1-2.101-114

UDK: 821.133.1.09Giono J.:1Nietzsche F.

\title{
La rencontre avec « un médecin philosophe »: une lecture du chapitre XIII du Hussard sur le toit de Jean Giono
}

\section{Daniela Curko}

\section{Résumé}

L'article étudie l'intertextualité de F. Nietzsche dans le chapitre XIII du roman Le hussard sur le toit de Jean Giono. Nous démontrons que le vieux médecin rencontré par Angelo à la fin de sa traversée d'une Provence ravagée par le choléra, est non seulement un maitre à penser, mais qu'il est ce "philosophe médecin de la culture » dont rêve Nietzsche. Par son diagnostic sur le mal qui est le choléra, le vieux praticien est effectivement redevable à la critique que Nietzsche fait de la civilisation occidentale et notamment du christianisme. Plusieurs preuves viennent étayer cette hypothèse, tout d'abord la représentation de notre civilisation comme décadente, accablée par la tristesse, et qui n'a rien à " offrir en échange » à la pulsion de mort ; la référence à la vie urbaine et à la grande ville comme lieu emblématique de la décadence ; enfin, la représentation du choléra comme « une vengeance de la chair qui raisonne » et qui se révolte contre le statut dévalorisant octroyé au corps, aux pulsions et à la pensée inconsciente dans notre civilisation.

Mots-clés : Giono, Nietzsche, civilisation, décadence, philosophe médecin de la culture 


\section{INTRODUCTION}

L'incipit du roman Le hussard sur le toit s'ouvre avec son héros, Angelo, déjà en route pour son voyage dans le sud de la Provence vers Gap afin d'accompagner, en preux chevalier, la jeune Pauline de Théus à son château de Gap ; ce récit narre la traversée d'Angelo qui voyage seul, puis en compagnie de Pauline, à travers une Provence ravagée par le choléra. Et si Angelo est appelé l'« homme de grand chemin ", c'est non seulement, comme le lui avait expliqué le marquis de Théus, " en l'honneur de l'amour du large et des vastes entreprises ", ${ }^{1}$ mais aussi parce qu'Angelo parcourt effectivement, tout au long du roman, les routes à l'aventure, ${ }^{2}$ et qu'il est donc, au sens métatextuel, le héros par excellence d'un roman d'aventures. Dans la clausule du roman, la fin de son périple et son arrivée à destination ne sont, pour Angelo, que le début d'un autre - vers l'Italie natale de ce carbonaro. Ce roman est avant tout celui d'un long voyage, interrompu par un long séjour à Manosque auquel le héros est contraint, puis par de courts arrêts dus à la claustration forcée, à l'emprisonnement provisoire du héros - à la quarantaine de la grange près des Omergues et à celle de Vaumeilh - ou bien à la fatigue, au besoin de sommeil, à la nécessité de s'abriter d'un orage...

Roman d'aventures donc, roman métaphysique et roman manichéen de la lutte du Bien contre le Mal, l'histoire d'Angelo est celle d'un voyageur et d'un voyage qui débute comme un voyage réel dans un espace-temps réel, puisque Angelo, parti du Sud de la Provence vers son Piémont natal, prend la direction du Nord et doit d'abord monter la montagne de Lure, une montagne des Préalpes de Haute-Provence. Pourtant, ce voyage réel se mue, dès les premières pages du roman, en voyage intérieur, véritable descente aux Enfers qui conduit le jeune homme pur et naif à travers la vision du Mal et la rencontre de ses méfaits sous la forme du choléra, vers son engagement dans cette bataille, puisque le héros apprend à agir contre le Mal. Il l'apprend grâce à ses initiateurs - personnages rencontrés sur son chemin et qui initient le jeune homme inexpérimenté au combat contre le choléra. Ainsi, «le petit français ", jeune médecin rencontré presqu'au début de sa route, à sa sortie des Omergues, premier village où Angelo voit la mort partout, lui a donné l'exemple de

1 Jean Giono, Angelo in Euvres romanesques complètes. Paris : Gallimard, 1977, t. IV, p. 120. Le marquis de Théus, époux de Pauline de Théus, héroïne du Husssard sur le toit, emploie cette expression dans un autre roman du cycle du Hussard, Angelo.

2 L'expression "l'homme de grand chemin » est donc polysémique. D'abord, elle en rappelle celle $\mathrm{du}$ " bandit, voleur de grand chemin », avec les connotations de la hardiesse, de l'impudence, de l'insolence; tout comme elle rappelle une expression très semblable, où le mot " chemin » est mis au pluriel : «être sur les grands chemins », qui signifie " aller à l'aventure, sans aucune idée de l'itinéraire et des événements qui peuvent survenir » (TFL), où, comme le précise le même dictionnaire, «l'accent est mis sur la notion de voyage aventureux » (Ibid.) 
l'abnégation, en lui apprenant à " chercher le dernier $»{ }^{3}$ donc à essayer, au prix de sa propre vie, de soigner et, pour se faire, de trouver, parmi les morts, celui qui est encore vivant et que l'on pourrait encore sauver. La vieille nonne du couvent abandonné de Manosque, quant à elle, l'investit d'une véritable mission en lui intimant l'ordre et le devoir de sauver les âmes des victimes du choléra, et à consoler leurs familles désemparées face à l'épidémie. Pourtant, ce n'est que vers la fin de son voyage qu'Angelo apprend à penser en psychologue, sociologue et finalement en philosophe, le Mal suprême qui est le choléra. Cette compréhension est rendue possible grâce au dernier initiateur d'Angelo, celui que Pauline et lui rencontrent par hasard, dans un village montagnard en ruines, alors qu'ils cherchent l'abri d'un vrai déluge, et qui semble être le dernier habitant d'un monde en ruine - un vieux médecin philosophe.

C'est ce vieil ermite qui propose à Angelo de suppléer la réflexion à l'action pour cerner et approcher ce mal qu'est le choléra - réflexion, il faut bien le dire, par endroits volontairement obscure et énigmatique, puisque Giono, dont le vieux médecin nous semble être un étrange porte-parole, dote celui-ci de son propre goût pour la lecture et la solitude, ainsi que pour la pipe ; l'auteur se plaît donc à égarer Angelo, et son lecteur, par un discours souvent décousu, à l'image du salon du vieux praticien ${ }^{4}$ où règne un désordre et un chaos inouïs :

Les flammes qui jaillissaient avec assez de force d'énormes bûches permettaient de voir l'énorme entassement de meubles très riches mais fort mal entretenus et tous surchargés de gros bouquins et de tas de papiers sur lesquels s'essayaient à l'équilibre des pichets, des brocs, des bols, des cuvettes, des bouteilles, des casseroles, des louches, des pipes de toutes les grosseurs, de toutes les formes et mêmes des tiroirs pleins d'ustensiles de cuisine. ${ }^{5}$

Et Angelo de se dépêtrer de ce bric-à-brac de pensées et de références sur le choléra, dont la chambre du vieux médecin est à la fois la métonymie et la métaphore.

Le vieux médecin est donc le dernier initiateur du novice Angelo, et représente une étape essentielle de son chemin initiatique, de sa compréhension de l'Homme, de la vie et surtout de la mort et du mal qu'est le choléra. Ce qui nous intéresse ici, c'est la pensée qui sous-tend son discours en apparence extravagant et incohérent, un discours surprenant, à la limite de l'incompréhensible. Qu'estce qu'Angelo, et le lecteur par-delà le personnage, apprennent sur le choléra de la bouche du vieux praticien?

3 Jean Giono, Le Hussard sur le toit in CEuvres romanesques complètes. Paris : Gallimard, 1977, p. IV, p. 280.

4 Cette chambre, encombrée de livres et de vieux papiers, ressemble d'ailleurs bien plus au bureau-bibliothèque d'un écrivain qu'au salon d'un praticien en retraite. 


\section{UN MÉDECIN PHILOSOPHE}

Lendroit où Angelo et Pauline rencontrent le personnage est déjà signifiant : en cherchant un abri pendant l'orage, ils arrivent dans un vieux village en ruine qui leur semble désert, et qui l'est en effet, à l'exception de ce vieux médecin, son dernier habitant. L'abandon, la déréliction et le délabrement du vieux village symbolisent l'état triste de décadence où se trouverait notre civilisation :

I1 [Angelo] était entré dans un débris de cave, ou de grange voûtée. Dehors, le déluge noyait les ruines d'un vieux village. [...]

Malgré l'épaisseur de la pluie et les nuages qui couraient à travers ce lieu élevé le village apparaissait complètement en ruine ; il ne restait que des chicots de murs. L'homme à la redingote leur fit traverser une sorte de place encombrée de buissons et d'herbes $[\ldots] .^{6}$

Le vieux médecin prévient Angelo dès le début en lui disant : "Je suis bien loin de vouloir vous faire un laïus : il ne s'agit pas du tout d'anatomie» (Ibid., p. 611). Car ce qu'il va proférer, malgré tout, c'est un laïus, mais d'une nature telle que cette étrange dissertation changera non seulement la vision que le héros a du choléra, mais surtout celle qu'il a du monde et de la société, puisque la maladie du corps est vue et représentée par le vieux médecin comme métaphore de l'état pathologique de la civilisation occidentale.

Le médecin, au lieu de parler, comme l'attendait Angelo, des traitements et des remèdes à administrer aux malades du choléra, donne en effet son diagnostic de l'état décadent de la civilisation de l'Europe contemporaine ${ }^{7}$ - et cette idée prédominante de la décadence, et de la tristesse de l'Homme des sociétés civilisées, de même que le reste du contenu de son discours que nous analyserons plus bas, en font ce " médecin philosophe $»^{8}$ de la culture, tant souhaité par Nietzsche. ${ }^{9}$ Car ce médecin constate que le choléra, mise à part une épidémie réelle, est surtout une des manifestations du malaise de notre civilisation. Dans un roman d'avant la Seconde Guerre Mondiale, Que ma joie demeure (paru en 1936), Giono avait d'ailleurs traité du même malaise, en le nommant

$6 \quad$ Ibid., p. 602-603.

7 Giono situe l'action de son roman en 1830-1832, lors de la grande épidémie de choléra en Provence.

8 Friedrich Nietzsche, Le Gai savoir. Paris : Flammarion, 2007, p. 29.

9 Anne-Marie Boisgontier a été la première à démontrer, dans sa thèse sur l'intertextualité nietzschéenne et l'éthique des personnages gioniens des Chroniques romanesques, que Giono a bien lu Nietzsche. Voir Anne-Marie Boigontier, Giono: par-delà le bien et le mal. Analyse de l'éthique, du bouleversement des valeurs dans les Chroniques. Thèse en microfiches : Caen, 1977. 
la « lèpre $»^{10}$ de l'ennui, et accablant de cette lèpre la communauté isolée du plateau de Grémone. Car la société occidentale ne peut pas offrir de perspective à l'Homme et ne lui donne plus de joie ; l'ennui est, d'après Giono, la véritable maladie de notre civilisation, qui fait des Hommes des «lépreux ${ }^{11}$ - ou des cholériques. Le choléra n'est, somme toute, qu'un symptôme ou une manifestation des plus spectaculaires de l'état décadent de notre civilisation, et Giono, à la suite de Nietzsche, évalue notre civilisation en termes de santé et de maladie, d'où la métaphore du philosophe médecin de la culture empruntée par Giono à la seconde préface du Gai savoir:

J'attends toujours qu'un médecin philosophe au sens exceptionnel du mot - un homme qui aura à étudier le problème de la santé d'ensemble d'un peuple, d'une époque, d'une race, de l'humanité - ait un jour le courage de porter mon soupçon à son degré ultime et d'oser cette proposition : dans toute activité philosophique, il ne s'agissait absolument pas jusqu'à présent de "vérité », mais de quelque chose d'autre, disons de santé, d'avenir, de croissance, de puissance, de vie ... ${ }^{12}$

\section{DIAGNOSTIQUER LE MALAISE DANS LA CIVILISATION}

Évidemment, le choléra, c'est aussi une des manifestations du Mal absolu qu'est la Shoah, puisque le vieux médecin fait une allusion au brassard jaune dont le port a été imposé aux Juifs par les Nazis et les gouvernements collaborationnistes, ${ }^{13}$ mais, mise à part une manifestation concrète du Mal à une époque historique donnée, le choléra est représenté par le vieux médecin comme un malaise intrinsèque et immanent à notre civilisation d'où la joie est exclue, selon un Giono lecteur de Nietzsche. Il faut dire que dans la pensée de Nietzsche, le terme même de « civilisation " a un sens particulier puisqu'il se réfère uniquement à notre civilisation contemporaine. Le terme a toujours un sens négatif pour Nietzsche, parce que, d'après lui, notre civilisation détruit ce qui est le meilleur en l'Homme en étouffant ses instincts au lieu de les sélectionner et de les discipliner (par l'élevage). En faisant cela elle fait aussi des ravages sur son esprit et tout penchant de celui-ci vers la grandeur. La civilisation est par définition ennemie de l'Homme supérieur, celui qui tend vers le Surhumain. En résumé : «La Civilisation, par exemple le christianisme, est une forme décadente de culture, qui ne met en œuvre qu'une

10 Jean Giono, Que ma joie demeure in Euvres romanesque complètes. Paris : Gallimard, t. II, p. 461.

11 Ibid., p. 469.

12 F. Nietzsche, Le Gai savoir, op. cit., p. 28.

13 «Actuellement, j'appelle choléra un brassard jaune et si je le fais porter à mille personnes, les milles crèvent en quinze jours. » (Le Hussard sur le toit, op. cit., 605) 
Zähmung ${ }^{14}$ [dressage] visant à brimer la volonté de puissance, à rendre malade et à affaiblir le type d'homme que Nietzsche caractérise comme supérieur, accompli, ou encore abouti, et à privilégier l'apparition d'un type décadent. $»^{15}$

Et si le vieux médecin exhorte Angelo d'être grec, ${ }^{16}$ c'est que la civilisation de l'ancienne Grèce est vue par Nietzsche comme l'antipode et l'antithèse de notre civilisation actuelle, comme la seule époque de la civilisation occidentale, avec la Renaissance italienne, où cette dernière ait atteint l'idéal de Kultur, d'une culture supérieure. Et cette « surabondance de santé, ce trop-plein de l'existence $»^{17}$ qui caractérise et distingue la société et la culture grecques dans la vision nietzschéenne, oppose la Grèce ancienne, époque de santé, à notre civilisation malade, exsangue, morose. Car l'Homme est dépourvu de joie dans notre civilisation, et Giono reprend ici sa métaphore des oiseaux de la joie qui s'envolent en laissant l'Homme seul, image à laquelle il a eu recours dans Que ma joie demeure ${ }^{18}$ :

I1 [le vieux médecin] voulait, disait-il, donner une description, même approximative, si je ne peux pas plus, hélas, de la façon dont enfin la conscience humaine se sentait alors dépouillée de toutes ses joies. Le souvenir même en est effacé. Il compara ces joies à des oiseaux. [...]

De toutes cette oisellerie et qui s'enfuit, non pas vers l'horizon mais vers le zénith, le ciel est plein, il en déborde. Il y en a tant qu'il en est embarrassé, que ses hauteurs s'engorgent, qu'il en souffre. ${ }^{19}$

Car dans cette vision, la civilisation occidentale moderne n'a rien à offrir à l'Homme, rien qui puisse satisfaire ses besoins d'épanouissement. Et le vieux médecin de dire avec ironie, en réponse à Angelo qui a remarqué que les villes manquent de chlore pour combattre le choléra :

Les villes ne manquent pas de chlorure, dit l'homme en allumant sa pipe. Elles manquent de tout; en tout cas de tout ce qu'il faut pour résister à une mouche, surtout quand cette mouche n'existe pas, comme c'est le cas. ${ }^{20}$

14 Le terme signifie « dressage » et désigne le mode de traitement des pulsions dans notre civilisation, qui vise à les brimer et à les éradiquer. Ce terme s'oppose donc à celui de l'élevage qui caractérise une Kultur [culture], terme désignant pour Nietzsche une culture supérieure.

15 Patrick Wotling, Nietzsche et le problème de la civilisation. Paris : PUF, 1995, p. 295.

16 «Soyez grec, mon jeune ami !» (Le Hussard sur le toit, op. cit., 608).

17 Friedrich Nietzsche, La naissance de la tragédie ou Hellénisme et pessimisme. Paris : Librairie Générale Française, 1994, p. 34.

18 Dans ce roman dédié tout entier à la problématique de l'ennui dans la civilisation moderne et à l'entreprise avortée, échouée de la joie, Giono montre la vieille fermière Marthe qui se désole du départ des oiseaux qui ont quitté son champ en hiver.

19 Jean Giono, Le Hussard sur le toit, op. cit., p. 616.

20 Ibid., p. 605. 
L'Homme, pour qui la vie n'a plus de valeur car il n'y a plus de joie, est en proie à l'instinct de mort, et Éros s'efface devant Thanatos. D'où le choléra, mal symbolique de l'Homme moderne. Chassez le naturel - le corps et sa pensée inconsciente, ses pulsions - il revient non seulement au galop mais il revient en cavalier de l'Apocalypse qui sème la mort :

Voici les premières lueurs du jour qui va peu à peu éclairer l'autre côté des choses. Le cholérique ne peut plus en détacher les regards. ${ }^{21}$

Le cholérique n'est pas un patient : c'est un impatient. Il vient de comprendre trop de choses essentielles. Il se hâte d'en connaître plus. ${ }^{22}$

Le meilleur, le seul remède ne serait pas d'administrer un médicament, une potion au malade, mais de lui redonner l'envie et le goût de vivre, d'imposer Éros au Thanatos, bref, « en un mot être plus fort, ou plus beau, ou plus séduisant que la mort. $» .{ }^{23}$ Ce savoir permettra plus tard à Angelo de sauver Pauline, quand la jeune femme tombera malade du choléra, et qu'il se mettra à frictionner son ventre par des gestes qui évoquent l'acte sexuel.

\section{LA CONDAMNATION DE LA VIE URBAINE}

Le vieux médecin se réfere à Paris comme un lieu d'où provient son état mélancolique :

I1 [le vieux médecin] avait exercé à Lyon, Grenoble et même Paris. C'était l'origine de sa mélancolie, dit-il avec ses lèvres dorées du plus délicat des sourires. (Ibid., p. 607)

Cette remarque du vieux médecin n'est pas anodine - il s'agit d'une condamnation sous-jacente et indirecte de la vie urbaine. Rappelons que Nietzsche et l'antimodernité qui s'inspire de sa pensée condamnent ensemble la vie urbaine et celle des grandes villes en particulier. L'image de la ville en général, et de la grande ville en particulier, pour Giono lecteur de Nietzsche, est corollaire du sens négatif du terme « civilisation» :

Quand la grande ville se transporte à la campagne, ce n'est pas de l'engrais qu'elle apporte aux champs, c'est de la pourriture et de l'horreur. ${ }^{24}$

La ville et la Grand-ville sont, pour le philosophe allemand, les lieux de la maladie emblématique de la civilisation moderne qu'est la dépression, conçue comme

21 Ibid., p. 618, nous mettons en italique.

22 Ibid., p. 618-619.

23 Ibid., p. 620.

24 Friedrich Nietzsche, La Volonté de puissance. Paris : Gallimard, 1995, t. II, p. 40. 
la conséquence de la destruction, par la civilisation moderne, de tout ce qu'il y a de meilleur en l'Homme. C'est pourquoi il dénonce la dépression comme étant le mal qui accable les habitants des villes dans la société industrialisée, elle est, selon lui, inhérente à la vie urbaine :

Crache sur cette ville des âmes déprimées et des poitrines étroites, des yeux perçants, des doigts poisseux $\left[\ldots . .{ }^{25}\right.$

Dans Ainsi parlait Zarathoustra, la ville péjorativement nommée « la Vache bariolée $»{ }^{26}$ représente toute ville de la civilisation moderne. Si toute ville de la civilisation moderne est le lieu de décadence, ou de la stagnation dans le meilleur des cas, une grande ville l'est encore plus que les autres villes, et Zarathoustra la fustige dans le chapitre «De passer son chemin $» .{ }^{27}$ Lieu emblématique de la civilisation moderne, la ville - et notamment la grande ville - porte toutes les tares dont le philosophe accuse la civilisation. Elle est en premier lieu la demeure de celui que Nietzsche voit comme le paradigme de l'Homme moderne - le Dernier Homme, l'Homme chez qui l'instinct, en soi naturel et sain, est bridé, brimé, asservi, dévié ou détruit ; l'Homme borné, médiocre, servile et hypocrite, incapable de quoi que ce soit de grand, l'Homme à l'esprit grégaire ${ }^{28}$ qui se complait dans le conformisme et l'autosatisfaction, l'antithèse du héros que tout Homme supérieur cache en lui. Le double de Zarathoustra, incarné par son fou, dépeint la Grand-ville en insistant sur l'image des abattoirs et sur celle du sang putride, ${ }^{29}$ métaphores que Giono reprend d'ailleurs dans sa description de la Villevieille du Chant du monde ${ }^{30}$ et de celle de la ville anonyme du Moulin de Pologne. ${ }^{31}$

25 Friedrich Nietzsche, Ainsi parlait Zarathoustra. Paris : Flammarion, 1996, p. 1996, p. 228.

26 «Vache bariolée» (en allemand bunte Kub) est une expression populaire ironique servant à désigner de petites agglomérations urbaines disparates dont la population serait dénuée de ce sens civique et politique qui fait la «Cité » ou la « Nation ». Pour reprendre la dérision du texte de Nietzsche, il faudrait traduire de façon irrévérencieuse par : "Trifouillis-les-Oies ».» (Note de Paul Mathias, in Friedrich Nietzsche, Ainsi parlait Zarathoustra, op. cit., 397).

27 Voir F. Nietzsche, Ainsi parlait Zarathoustra, op. cit., p. 226-230.

28 Le fou de Zarathoustra décrit l'esprit grégaire du Dernier Homme de la manière suivante : « Pas de berger et un seul troupeau! » (Ibid., p. 53)

29 «Ici pourrissent tous les grands sentiments ; ici l'on n'autorise que le cliquetis de menus sentiments entièrement décharnés. [...]

Ne sens-tu pas l'odeur des abattoirs et des gargotes de l'esprit ? Cette ville n'exhale-t-elle pas un fumet d'esprit massacré ? [...]

Ici le sang coule putride, tiède et spumeux dans toutes les veines; crache sur la Grand-ville, sur cette grande sentine où fermentent toutes les lies. »(Ibid., p. 227, nous mettons en italique).

30 Voir Jean Giono, Le Chant du monde in CEuvres romanesques complètes. Paris : Gallimard, 1972, t. II, p. 275-276, 283.

31 Voir Jean Giono, Le Moulin de Pologne in CEuvres romanesques complètes. Paris : Gallimard, 1980, t. V, p. 694. 


\section{DEVENIR LION}

L'image du lion brodé, réitérée deux fois, est le moyen par lequel le vieux médecin semble parler du cœur, au sens de « courage ». Elle n'a vraiment de sens qu'interprétée dans le contexte de la pensée nietzschéenne :

Non, mademoiselle, je n'ai pas parlé de cœur : ouvrage de dame. C'est un lion que nous portons brodé sur la chemise. [...]

Vous caressez subrepticement le lion brodé sur votre chemise. ${ }^{32}$

Or, dans le contexte de l'interprétation insolite qu'entreprend le médecin du phénomène du choléra - il la représente, nous l'avons vu, comme une manifestation, certes effrayante, du mal inhérent dans notre civilisation occidentale - il semble que le vieux médecin appelle indirectement à la révolte, ou au moins à un besoin de remise en question de notre civilisation et de la société occidentales, et à un besoin de réévaluation de toutes les valeurs (le terme de réévaluation est d'ailleurs un des termes clés de la pensée de Nietzsche). Car l'image du lion symbolise chez Nietzsche précisément la révolte contre les anciennes valeurs qui entravent $1^{\prime} H_{0} \mathrm{~m}^{33}$ et dont il faut s'affranchir afin de réaliser une culture, une culture supérieure, saine par définition.

\section{LE CHOLÉRA, VENGEANCE DE LA CHAIR QUI RAISONNE}

Le choléra, ainsi que le médecin l'explique à Angelo, est une sorte de revanche du corps trop longtemps opprimé par la raison. Notre civilisation accorde à celle-ci la primauté et un statut ontologique supérieur. Le choléra devient ainsi une étrange critique de la raison pure :

Entrez, entrons dans ces cinq à six pieds cubes de chair qui va devenir cholérique, de chair en proie au prodrome de ce cancer de la raison pure, de chair fatiguée des détours que lui fait prendre sa matière grise, qui raisonne soudain à l'aide de ses mystères et met les bouchées doubles. ${ }^{34}$

32 Le Hussard sur le toit, op. cit., p. 614.

33 Rappelons qu'à la différence du chameau, symbole de l'homme qui, plein de respect et de déférence pour les valeurs millénaires, n'en est que le continuateur docile et obéissant, le lion est celui qui s'en libère, afin de pouvoir par la suite devenir créateur de nouvelles valeurs, cette créativité étant symbolisée par l'image de l'enfant, la troisième et dernière métamorphose. Voir Nietzsche, Ainsi parlait Zarathoustra, op. cit., 63-65.

34 Ibid., p. 616. 
En effet, dans la pensée de Nietzsche, la valorisation du corps et sa pensée inconsciente ont pour corolaire la dévalorisation de la conscience..$^{35}$ Le corps, pour Nietzsche, est d'abord pensé comme structure pulsionnelle et sa fonction est primordiale dans le processus d'interprétation. Selon le philosophe, le corps est considéré comme le siège du Soi ${ }^{36}$ de la pensée inconsciente à laquelle Nietzsche accorde plus de valeur qu'à la conscience. Nietzsche considère en effet celle-ci comme « la dernière et la plus tardive évolution de l'organique et par conséquent aussi ce qu'il y a en lui de plus inachevé et du moins solide. ${ }^{37}$

\section{LES LOUANGES DU CORPS ET DE LA TERRE}

Si le choléra est, comme l'explique le vieux médecin, le mal de la « chair qui raisonne $",{ }^{38}$ parce que la civilisation a trop longtemps opprimé le corps et méprisé la pensée inconsciente, on ne peut la vaincre qu'en libérant ce corps et en le mettant en valeur par l'élevage des pulsions qui ne sont point brimées. Et le vieux médecin de souligner la beauté du corps d'Angelo. À plusieurs reprises il exhorte le jeune homme à abandonner sa pudeur, afin de célébrer et de libérer ce corps dont une des raisons d'être est précisément d'aimer :

Au diable la pudeur, lui dit l'homme, restez là près du feu. Vous êtes bien fait, qu'est-ce que vous risquez ? Croyez-vous que Mademoiselle a été créée et mise au monde per studiare la matematica? [...] (Ibid., p. 608)

D'ailleurs, la passion d'Angelo et de Pauline, sentiment qui ne s'exprime guère à cause de l'interdit de l'adultère, n'échappe point à l'œil perspicace du vieux praticien qui semble inciter les deux jeunes à vivre leur passion sans culpabilité en leur représentant celle-ci comme une évidence. Le médecin philosophe de Giono,

35 Nietzsche inverse la hiérarchie des valeurs installée depuis longtemps dans notre civilisation en mettant le corps en haut et la raison en bas, puisque le corps n'est pas que le corps - il s'inscrit dans la perspective interprétative : "La conscience est la dernière et la plus tardive évolution de l'organique et par conséquent aussi ce qu'il y a en lui de plus inachevé et du moins solide. " (Nietzsche, Le Gai savoir, op. cit, p. 69). Comme le remarque Karl Jaspers, le corps est dans sa pensée le sujet des fonctions inconscientes :

"Nietzsche appelle la forme et la vie de l'homme : son corps. Celui-ci n'est pas le corps purement anatomique, non point le cadavre, mais le tout des vivantes fonctions inconscientes, dans lesquelles nous sommes englobés. » (Karl Jaspers, Nietzsche. Introduction à sa philosophie. Paris : Gallimard, 1950, p. 317).

36 «Par-delà tes pensées et tes sentiments, mon frère, il y a un maître puissant, un sage inconnu, qui s'appelle le Soi. Il habite ton corps, il est ton corps. Il y a plus de raison dans ton corps que dans l'essence même de ta sagesse. » (Nietzsche, Ainsi parlait Zarathoustra, op. cit., 72).

37 Nietzsche, Le Gai savoir, op. cit., p. 69.

38 Le Hussard sur le toit, op. cit., p. 616. 
réalisation romanesque de l'idéal du médecin philosophe de Nietzsche, n'omet pas de représenter le désir et l'attachement qui unissent Angelo et sa compagne de voyage, comme quelque chose à la fois de bon, d'acceptable et même de très naturel, comme s'il chantait, lui aussi, « les louanges du corps et de la terre ».39

\section{CONCLUSION}

Nous avons vu que le vieux médecin philosophe était donc le premier et le seul des personnages rencontrés par Angelo lors de sa traversée d'un pays dévasté par le choléra qui ait permis à Angelo de penser le mal que représente cette épidémie. Or, nous avons souligné que le vieux médecin le faisait - et Giono ici avait déployé tout son sens de l'humour - dans un discours qui n'en était pas un, tant il était décousu, chaotique, à la limite du compréhensible. Dans ce coq-à-l'âne du vieux solitaire, plein d'allusions énigmatiques, ni Angelo ni le lecteur ne trouveront pas d'explication scientifique de l'épidémie, ce dont le vieux médecin avait d'ailleurs explicitement prévenu Angelo. Ils trouveront, par contre, dans ce qui semble être une divagation du vieil original une sorte de résumé, certes délibérément très confus et lacunaire, de la critique que fait Nietzsche de la civilisation occidentale et notamment du christianisme. Le choléra est, pour le vieux praticien, symptôme d'un malaise très profond, d'une crise de civilisation où le terme de civilisation a le même sens - uniquement négatif, qu'il a chez Nietzsche pour qui la Civilisation est opposée à la culture. Alors que cette dernière représente l'idéal d'une haute culture, telle qu'a été la civilisation de la Grèce antique ou de la Renaissance, la Civilisation (qui est donc notre civilisation occidentale) opprime les instincts, les dresse au lieu de les élever - au lieu d'apprendre aux hommes à les dominer, tout comme elle dénigre et déprécie le corps. D'où, d'après Nietzsche, la dépression qui n'est donc qu'une manifestation du désir de mort et du nihilisme passif, « sentiment creusant de rien ${ }^{40}$ omniprésent dans notre civilisation.

Pour Nietzsche, le corps n'est pas uniquement une entité physique, mais il est avant tout une structure très complexe de pulsions et le siège de la pensée inconsciente qu'il s'agit d'explorer et de revaloriser. Et le désir est une de ses pulsions, et d'une grande importance pour tout être humain. D'où l'accent que met le vieux praticien de Giono sur le désir physique, son incitation à la prise de conscience

39 "Loyal entre tous, ce Moi parle du corps et veut le corps, même quand il rêve et divague ou papillonne, les ailes brisées.

Ce Moi apprend à s'exprimer avec une loyauté croissante ; et mieux il l'apprend, plus il trouve de mots pour dire les louanges du corps et de la terre. » (Nietzsche, Ainsi parlait Zarathoustra, op. cit., p. 70)

40 Friedrich Nietzsche, Fragments posthumes in Oeuvres philosophiques completes, Paris : Gallimard, 1968-1997, t. XI, p. 228. 
d'Angelo du désir inavoué et inavouable qu'il ressent pour Pauline, femme à ses yeux interdite car épouse digne, chaste et fidèle du vieux marquis de Théus.

Par la représentation, certes lapidaire, de la grande ville - notamment de Lyon - comme le lieu emblématique d'une civilisation décadente Giono est également redevable à Nietzsche.

\section{Bibliographie}

Barbarić, Damir. „Friedrich Nietzsche“, in : Suvremena filozofija I [éd. Ozren Žunec]. Zagreb : Školska knjiga, 1996, pp. 343-371.

Boigontier, Anne-Marie. Giono : par-delà le bien et le mal. Analyse de l'éthique, du bouleversement des valeurs dans les Chroniques. Th : Litt. : Caen, 1977.

Ćurko, Daniela. «Un grand arbre et un homme supérieur: l'étude de l'intertexte nietzschéen dans Un roi sans divertissement de Jean Giono », in: Studia romanica et anglica zagrabiensia, n. 57/2012, pp.3-30. ISSN: 0039-3339.

Ćurko, Daniela. « L'utopie dans les romans antimodernistes de J. Giono et l'intertexte nietzschéen », in : Rencontres Jules Verne. Sciences, crises et utopie (Actes des Rencontres Jules Vernes, 22-23 nov 2013 - École Centrale, Nantes), éd. Philippe Mustière et Michel Fabre, Nantes : éd. Coiffard, pp. 128-136. ISBN 978-2919339-22-8.

Deleuze, Gilles. Nietzsche et la philosophie. Paris : Presses Universitaires de France, 1962.

Fink, Eugen. Nietzscheova fllozofija. Zagreb : Centar za kulturnu djelatnost, 1981. Giono, Jean. Angelo. In : Oeuvres romanesques complètes, tome IV, Paris : Gallimard, 1977.

Giono, Jean. Le Chant du monde. In : Oeuvres romanesques complètes, tome II, Paris : Gallimard, 1972.

Giono, Jean. Le Hussard sur le toit. In : Oeuvres romanesques complètes, tome IV, Paris : Gallimard, 1977.

Giono, Jean. Le Moulin de Pologne. In : Oeuvres romanesques complètes, tome V, Paris : Gallimard, 1980.

Giono, Jean. Que ma joie demeure. In : Oeuvres romanesques complètes, tome II, Paris : Gallimard, 1972.

Granier, Jean. Nietzsche, Paris : PUF, 1982.

Heidegger, Martin. Nietzsche [trad. en anglais par David Farrell Krell]. San Francisco: éd. HarperCollins, 1991.

Heidegger, Martin. Nietzsche [trad. en français par Pierre Klosowski]. Paris : Gallimard, 1971.

Heidegger, Martin. Nietzscheova metafyzika [trad. de l'allemand en croate par Šime Vranić]. Zagreb : Visovac, 1994. 
Jaspers, Karl. Nietzsche. Introduction à sa philosophie [trad. de l'allemand par Henri Niel]. Paris : Galliemard, 1950.

Le Trésor de la langue française. CNRS/Université de Lorraine. Adresse : http:// atilf.atilf.fr.

Mathias, Paul. Présentation d'Ainsi parlait Zarathoustra, in : Friedrich Nietzsche. Ainsi parlait Zarathoustra, Paris : Flammarion, 1996, pp. 9-39.

Nietzsche, Friedrich. Ainsi parlait Zarathoustra [trad. en français par Geneviève Bianquis], Paris : Flammarion, 1996.

Nietzsche, Friedrich. Le Gai savoir [trad. par Patrick Wotling]. Paris : Flammarion, 2007.

Nietzsche, Friedrich, Filozofija u tragičnom dobu Grka [trad. en croate par Petar Milat]. Zagreb : Naklada Jesenski i Turk, 2001.

Nietzsche, Friedrich. Fragments posthumes in : Euvres philosophiques complètes, t. IX-XIV, Paris : Gallimard, 1968-1997.

Nietzsche, Friedrich. La Naissance de la tragédie ou Hellénisme et Pessimisme [trad. par Jean Marnold et Jacques Morland]. Paris : Librairie Générale française, 1994.

Nietzsche, Friedrich. La Volonté de puissance I. Paris : Gallimard, 1995.

Nietzsche, Friedrich. Volonté de puissance II. Paris : Gallimard, 1995.

Wotling, Patrick. "Affectivité et valeurs ", in Lectures de Nietzsche (sous la dir. de Jean-François Balaudé et Patrick Wotling). Paris : Le livre de poche, 2000, pp. 131-160.

Wotling, Patrick. Le vocabulaire de Nietzsche. Paris : Ellipses, 2001.

Wotling, Patrick. 1995. Nietzsche et le problème de la civilisation. Paris : PUF, 1995.

Daniela Ćurko Université de Zadar, Croatie dcurko@unizd.hr

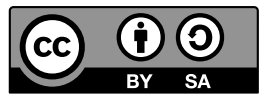

\section{Srečanje z »zdravnikom filozofom«: branje 13. poglavja knjige Hus- sard sur le toit Jeana Gionoja}

Prispevek analizira intertekstualnost F. Nietzscheja v 13. poglavju romana Le bussard sur le toit. Stari zdravnik, ki ga sreča Angelo, ni le mislec, ampak predstavlja tudi »filozofskega zdravnika kulture« o katerem sanja Nietzsche.

Ključne besede: Giono, Nietzsche, civilizacija, dekadenca, filozofski zdravnik kulture 
The Meeting with the Physician Philosopher. Reading Chapter 13 of the Novel Le hussard sur le toit by Jean Giono

The article analyzes the intertextuality of F. Nietzsche in Chapter 13 of the novel Le hussard sur le toit. The old physician Angelo meets is not only a thinker, but also represents a philosophical doctor of culture about whom Nietzsche dreams.

Keywords: Giono, Nietzsche, civilisation, decadence, a philosophical physician of culture 